PHYSICAL REVIEW D 78, 089902(E) (2008)

\title{
Erratum: Causal hydrodynamics of gauge theory plasmas from AdS/CFT duality [Phys. Rev. D 77, 066014 (2008)]
}

\author{
Makoto Natsuume and Takashi Okamura \\ (Received 30 September 2008; published 13 October 2008)
}

DOI: 10.1103/PhysRevD.78.089902

PACS numbers: 11.25.Tq, 12.38.Mh, 99.10.Cd

The perturbative solution for the shear mode reported in our paper referenced in the title is incorrect. The solution (B1)(B4) should be

$$
\begin{gathered}
F_{0}=C, \quad F_{1}=i C\left(-\frac{1}{u}+\ln (1+u)\right), \quad G_{1}=\frac{C}{2 u}, \\
F_{2}=C\left[\frac{-1+\ln 2}{u}+\frac{\ln 2}{2} \ln (u-1)-\frac{1}{4} \ln (u+1) \ln \left\{(1-u)^{2}(u+1)\right\}-\frac{1}{2} \operatorname{Li}_{2}\left(\frac{u+1}{2}\right)\right], \\
H_{11}=-i C\left[\frac{1}{u}\left(1-\frac{1}{2} \ln (u+1)\right)+\ln \left(\frac{u}{u+1}\right)\right], \\
G_{2}=\frac{1}{2} C\left[\frac{1}{u}+\ln \left(\frac{u}{u+1}\right)\right] .
\end{gathered}
$$

The solution given in the paper is actually the perturbative solution for the diffusive mode although $F_{1}$ is incorrect. The solution $F_{1}$ for the diffusive mode should be

$$
F_{1}=C \ln \frac{1+u}{2 u}
$$

